\title{
RATES OF TUBERCULOSIS INFECTION IN Healthcare Workers Providing Services to HIV-INFECTED POPULATIONS
}

Karen Zahnow, RN, MEd; John P. Matts, PhD; David Hillman, MS; Elizabeth Finley, RN, ANP; Lawrence S. Brown, Jr, MD, MPH;

Ramon A. Torres, MD; Jerome Ernst, MD; Wafaa El-Sadr, MD, MPH; George Perez, MD; Carol Webster, RN, MPH; Beverly Barber, RN; Fred M. Gordin, MD; the Terry Beirn Community Programs for Clinical Research on AIDS

\begin{abstract}
OBJECTIVE: To assess the prevalence of tuberculosis (TB) or a positive skin test in healthcare workers (HCWs) providing services to human immunodeficiency virus (HIV)-infected individuals and to determine prospectively the incidence of new infections in this population.

DESIGN: This prospective cohort study enrolled 1,014 HCWs working with HIV-infected populations from 10 metropolitan areas. Purified protein derivative (PPD) tuberculin skin tests were placed at baseline and every 6 months afterwards on those without a history of TB or a positive PPD. Demographic, occupational, and TB exposure data also were collected.

SETTING: Outpatient clinics, hospitals, private practice offices, and drug treatment programs providing HIV-related healthcare and research programs.

PARTICIPANTS: A voluntary sample of staff and volunteers from 16 Community Programs for Clinical Research on AIDS units.

RESULTS: Factors related to prior TB or a positive skin test at baseline included being foreign-born, increased length of time in health care, living in New York City, or previous bacille Calmette-Guerin vaccination. The rate of PPD conversion was 1.8 per 100 person years of follow-up. No independent relation was found between the amount or type of contact with HIV-infected populations and the risk of TB infection.

CONCLUSION: These data provide some reassurance that caring for HIV-infected patients is not related to an increased rate of TB infection among HCWs in these settings (Infect Control Hosp Epidemiol 1998;19:829-835).
\end{abstract}

As tuberculosis (TB) case rates have increased during the past 10 years, many concerns have been raised about the risk of acquired $\mathrm{TB}$ infection among healthcare workers (HCWs). ${ }^{1,2}$ Recent reports of TB outbreaks in healthcare and prison settings have fueled this concern, especially with the documentation of multidrug-resistant TB occurring among exposed HCWs. ${ }^{3-5}$ Some individuals have questioned whether working primarily with those infected with human immunodeficiency virus (HIV) increases an individual HCW's risk of acquiring TB. Factors supporting this possibility include the increased potential for exposure to patients with active TB, plus the difficulties encountered in diagnosing TB in an HIV-infected person. ${ }^{4-8}$ However, an increased level of awareness among HCWs in these settings and the

From the Division of Infectious Diseases (Dr. Gordin and Ms. Zahnow, Ms. Finley), Veterans' Affairs Medical Center, Washington, DC; the Division of Biostatistics (Dr. Matts, Mr. Hillman), School of Public Health, University of Minnesota, Minneapolis, Minnesota; Addiction Research and Treatment Corporation (Dr. Brown) and Harlem Hospital, Columbia University, New York City; Clinical Directors' Network of Region II and St Vincent's Hospital and Medical Center of New York (Dr. Torres), New York City; Bronx-Lebanon Hospital Center (Dr. Ernst), Bronx, New York; the Division of Infectious Diseases (Dr. El-Sadr), Harlem Hospital Center, New York City, New York; New Jersey Community Research Initiative and Atlantic City Medical Center (Dr. Perez), New Jersey; Richmond AIDS Consortium (Ms. Webster), Richmond, Virginia; Denver CPCRA (Ms. Barber), Denver Public Health Department, Denver, Colorado.

The authors are indebted to their collaborators at the following participating study units: Clinical Directors Network of Region II-Linda Podhurst, PhD, Garance Franke-Ruta, and Valerie Zbikowski; Bronx-Lebanon Hospital Center-Lena McDowell, Daya Koyande, MD, Bruce Soloway, MD; Harlem AIDS Treatment Group-Cheryl Guity, RN, Michelle Hardy, BA, and Susan Caras, RN; New Jersey Community Research Initiative-Jean Haspel-Brooks, RN, BSN, Ann Marie Sheridan, RN, BSN, and Victoria Taylor, RN; Richmond AIDS Consortium-Thomas Kerkering, MD, Patricia $S$. Bragg, RN, MPH, and Margaret D. Britton, RN, BSN; Denver Community Programs for Clinical Research on AIDS (CPCRA)-Michael Grodesky, RN, MS, ANP, Joan Davis, RN, MS, MPA, and Jack Rouff, BS, MSW, MBA; Hill Health Center-Robin Vogel, PA-C, Beryl Harper, RN, and Delia Santana, CHW; Addiction Research and Treatment Corporation-Ansiret Suza, RPA-C, and Stanley John, MD; Henry Ford Hospital-Diane MastroPolak, RN, Louis Saravolatz, MD, and Bishe Al-Vjayli, MD; Louisiana Community AIDS Research Program-Sister Sue Pablovich, RN, NP, MPH, Janice Walker, RN, MN, and Suzanne LeBlanc, RN; Wayne State University-Lawrence R. Crane, MD, Lorraine Neubauer, RN, BSN, and Joette Lambert, RN, BSN; AIDS Research Consortium of Atlanta-Melanie Thompson, MD, Ken Mabry, MBA, and Betty Mear, RN; Delaware CPCRASharon Chanpimol, BSN, Karen Swanson, BSN, RN, CCRC, Arlene Bincsik, MS, RN, CCRC; Research and Education Group-Jim Sampson, MD, Karen Sepich, RN, and David Fleming, MD; Chicago CPCRA-Roberta Luskin-Hawk, MD, Renee Krupa, RN, and Roger Sullivan, RN.

Address reprint requests to Fred M. Gordin, MD, Infectious Diseases (2C South), VA Medical Center, 50 Irving St NW, Washington, DC 20422.

97-OA-140. Zahnow K, Matts JP, Hillman D, Finley E, Brown LS Jr, Torres RA, Ernst J, El-Sadr W, Perez G, Webster C, Barber B, Gordin FM, the Terry Beirn Community Programs for Clinical Research on AIDS. Rates of tuberculosis infection in healthcare workers providing services to HIVinfected populations. Infect Control Hosp Epidemiol 1998;19:829-835. 
implementation of appropriate infection control procedures may offset this risk.

Few prospective studies have been done to assess the risk of acquired TB among HCWs in any setting, as measured by purified protein derivative (PPD) tuberculin skin-test conversions. One study of medical students in Chicago found a conversion rate of $5 \%$ during their third year of medical school. ${ }^{9}$ Retrospective studies have found baseline prevalence rates of positive PPDs among $\mathrm{HCWs}$ as high as $50 \%$ and conversion rates after a known exposure as high as $76 \% .^{10}$ Prior to implementing expanded infection control guidelines for TB in the early 1990s, Grady Memorial Hospital in Atlanta had 1-year conversion rates of $13.2 \%$ for nursing personnel and over $6 \%$ for HCWs in general. ${ }^{11}$ Little has been documented about the risk of TB infection to HCWs working with HIV-infected patients, and even less is known about the characteristics of the HCWs themselves that may influence the prevalence and incidence of TB infection or disease in this group.

To understand the risk of TB in HCWs working with HIV-infected patients better, we undertook this study to assess the prevalence of TB or a positive skin test and to determine prospectively the incidence of new infections in this population of HCWs.

\section{METHODS}

The Terry Beirn Community Programs for Clinical Research on AIDS is a National Institutes of Health-funded network of 17 units (hospitals, clinics, and private physicians) across the United States that provides care to HIVinfected persons, with a special emphasis on injecting drug users, people of color, and women. The staff members and volunteers at 16 of these units were recruited to participate in this observational study of the baseline prevalence of TB or a positive PPD test and the prospective collection of tuberculin skin-test conversion data. Each unit submitted information about the number of patients seen with HIV or TB, and individual HCWs were recruited between September 1992 and May 1993. All HCWs completed a questionnaire that included basic demographic information, job title and description, past and ongoing exposure to TB, and history of work in the healthcare field. Each person also was requested to complete an HIV status questionnaire, which could be completed and submitted anonymously. Participants could identify themselves as being HIV-positive, HIV-negative, unaware of HIV status, or unwilling to answer the question. Information on race and gender was not collected deliberately to protect confidentiality further.

Participants who had no history of prior TB or a positive skin test received a baseline test upon entering the study, using 5 TU of PPD given intradermally using the Mantoux technique. These individuals continued to receive a PPD skin test every 6 months until the close of the study (July 1995), as long as their test remained negative. Twostep skin testing was recommended but not required at baseline for anyone who had not received a PPD in the past 2 years. In accordance with published guidelines, ${ }^{12}$ a 10 $\mathrm{mm}$ or greater induration at 48 to 72 hours was considered positive unless the participant was known to be HIVpositive or had been in recent close contact with someone diagnosed with $\mathrm{TB}$, in which case a 5 -mm or greater induration was considered positive. Clinical decisions about TB prophylaxis were left to the discretion of the participants and their physicians. Information about possible TB exposure in the community, job responsibilities, and percentage of current caseload known to be HIV-infected or to have active TB also was collected at each visit.

Chi-square tests and chi-square tests for linear trend were used to assess categorical data. Data tabulated as not applicable, unknown, or missing were not included in the calculation of test statistics or $P$ values. Stepwise logistic regression was used to identify factors independently related to TB infection. Inclusion of variables was stopped when no other variables would be statistically significant. Additional analyses were done using classification and regression trees ${ }^{13}$ to explore the data for relationships not identified by the logistic regression (because no important relationships other than those identified in the logistic regression were found, only the logistic regression results are reported). A Kaplan-Meier approach was used to estimate cumulative PPD conversion rates, and a Poisson regression was used to compare incidence rates among subgroups. A two-sided $P$ less than .05 was considered statistically significant. No adjustments were made for multiple comparisons.

\section{RESULTS}

A total of 1,014 HCWs were enrolled in the study from 16 clinical units, and their demographic and background data are provided in Table 1. The greater New York City (NYC) area was composed of 6 units, including one each from northern New Jersey and southern Connecticut. The 10 non-NYC units were located in Atlanta; Chicago; Denver; Detroit; New Orleans; Portland, Oregon; Richmond, Virginia; Washington, DC; and Wilmington, Delaware. Each participating clinical unit was composed of one or more subsites. There were 57 sites, of which 56 provided site characteristic data. The site with the missing data had only one HCW in the study. The research interests of this consortium are reflected in the high percentage of HIV-infected patients seen at these sites, although each study participant saw variable proportions of HIV-infected patients, depending on job responsibilities and site characteristics (eg, drug treatment programs).

Additional data on the study participants include that almost half averaged 10 or more hours per week of direct hands-on care of patients, including physical examinations, nursing care, and treatments. Eighty-one were both foreignborn and had a history of bacille Calmette-Guerín (BCG) vaccination. The HIV status questionnaire was returned by 746 (74\%) of the participants. Of those responding, $15(2 \%)$ self-reported as HIV-positive, 505 (68\%) as HIV-negative, $210(28 \%)$ as unaware of their HIV status, and $16(2 \%)$ chose not to answer the question yet returned the form.

At the time of enrollment, 209 (21\%) of the HCWs 
TABLE 1

Relation of Demographic and Occupational Characteristics in 1,014* Healthcare Workers Defined as Positive at EnTrY

\begin{tabular}{|c|c|c|c|c|}
\hline \multirow{2}{*}{$\begin{array}{l}\text { Characteristic } \\
\text { Age }\end{array}$} & \multicolumn{2}{|c|}{ No. (\%) } & \multirow[t]{2}{*}{ Percentage Posittve at Study Entry } & \multirow[t]{2}{*}{$\boldsymbol{P}$} \\
\hline & & & & \\
\hline$<30$ & 190 & (18.8) & 17.4 & $<.001$ \\
\hline $30-39$ & 413 & $(40.9)$ & 22.5 & \\
\hline $40-49$ & 275 & (27.3) & 26.2 & \\
\hline$\geqslant 50$ & 131 & $(13.0)$ & 35.9 & \\
\hline \multicolumn{5}{|l|}{ Area of country } \\
\hline NYC area & 546 & $(53.8)$ & 31.1 & $<.001$ \\
\hline Non-NYC area & 468 & $(46.2)$ & 16.7 & \\
\hline \multicolumn{5}{|c|}{ Foreign-born (or resided $>1 \mathrm{y}$ in endemic area) } \\
\hline Yes & 214 & $(21.1)$ & 51.4 & $<.001$ \\
\hline No & 800 & $(78.9)$ & 17.3 & \\
\hline \multicolumn{5}{|l|}{ BCG vaccination in past } \\
\hline Yes & 105 & $(10.4)$ & 75.2 & $<.001$ \\
\hline No & 805 & $(79.4)$ & 16.3 & \\
\hline Uncertain or missing & 104 & $(10.3)$ & 36.5 & \\
\hline \multicolumn{5}{|l|}{ Occupation type } \\
\hline Physicians & 182 & $(18.0)$ & 34.1 & .002 \\
\hline PAs, NPs, or nurses & 264 & $(26.1)$ & 27.3 & \\
\hline Social workers or counselors & 134 & $(13.2)$ & 21.6 & \\
\hline Clerical & 155 & $(15.3)$ & 17.4 & \\
\hline Other & 278 & $(27.4)$ & 20.5 & \\
\hline \multicolumn{5}{|l|}{ Years in heath care } \\
\hline$<5$ & 249 & (26.3) & 13.7 & $<.001$ \\
\hline $5-9$ & 204 & (21.5) & 24.0 & \\
\hline $10-14$ & 203 & $(21.4)$ & 23.2 & \\
\hline$\geqslant 15$ & 292 & $(30.8)$ & 31.8 & \\
\hline \multicolumn{5}{|l|}{ Years worked with HIV-infected patients } \\
\hline$<5$ & 519 & $(55.1)$ & 21.8 & .105 \\
\hline $5-9$ & 305 & $(32.4)$ & 25.3 & \\
\hline$\geqslant 10$ & 118 & $(12.5)$ & 28.0 & \\
\hline \multicolumn{5}{|l|}{ Proportion of patients with HIV infection } \\
\hline $0 \%-24 \%$ & 203 & $(20.0)$ & 19.7 & $.141^{\dagger}$ \\
\hline $25 \%-49 \%$ & 98 & $(9.7)$ & 27.6 & \\
\hline $50 \%-74 \%$ & 155 & (15.3) & 25.2 & \\
\hline $75 \%-100 \%$ & 438 & $(43.2)$ & 26.0 & \\
\hline Unknown, not applicable, or missing & 120 & $(11.8)$ & 23.3 & \\
\hline \multicolumn{5}{|l|}{ Proportion of patients with active TB } \\
\hline $0 \%-24 \%$ & 683 & $(67.4)$ & 23.3 & $<.001^{\dagger}$ \\
\hline $25 \%-49 \%$ & 57 & $(5.6)$ & 36.8 & \\
\hline $50 \%-74 \%$ & 25 & (2.5) & 44.0 & \\
\hline $75 \%-100 \%$ & 14 & (1.4) & 42.9 & \\
\hline Unknown, not applicable, or missing & 235 & $(23.2)$ & 21.7 & \\
\hline \multicolumn{5}{|l|}{ Performs any cough-inducing procedures?‡ } \\
\hline Yes & 316 & (31.2) & 31.7 & $<.001$ \\
\hline No & 698 & $(68.8)$ & 21.2 & \\
\hline \multicolumn{5}{|l|}{ Current occupational setting } \\
\hline Outpatient & 265 & $(26.2)$ & 17.7 & $<.001$ \\
\hline Hospital-based & 554 & $(54.7)$ & 28.9 & \\
\hline Private practice & 89 & $(8.8)$ & 13.5 & \\
\hline Drug treatment program & 74 & (7.3) & 29.7 & \\
\hline Other & 31 & (3.1) & 22.6 & \\
\hline
\end{tabular}

Abbreviations: BCG, bacille Calmette-Guerin; HCW, healthcare worker; HIV, human immunodeficiency virus; NP, nurse practitioner; NYC, New York City; PA, physician assistant; TB, tuberculosis. * Certain data missing for some HCWs.

† Chi-square test for trend.

¥ Bronchoscopies, endoscopies, sputum collection, administration of aerosols. 


\section{TABLE 2}

Multivariate ANalysis of History of Tuberculosis or a Posttive Purified Protein Derivative in 805 HealthCare Workers Who Did Not Have a Prior Bacille CalmetteGUERÍN VACCINATION

\begin{tabular}{lccc}
\hline Predictor & OR & CI $_{9 \mathbf{5}}$ & $\boldsymbol{P}$ \\
\hline New York City area & 1.82 & $(1.21-2.74)$ & .004 \\
Foreign-born & 2.07 & $(1.23-3.49)$ & .006 \\
Years as HCW (5-year periods) $^{*}$ & 1.37 & $(1.23-1.52)$ & $<.0001$ \\
\hline
\end{tabular}

Abbreviations: $\mathrm{Cl}_{95}, 95 \%$ confidence interval; $\mathrm{HCW}$, healthcare worker; $\mathrm{OR}$, odds ratio. * For every 5-year increment, the odds ratio increases $37 \%$

had a history of TB or a history of a positive tuberculin skin test. Information about time of PPD positivity, time of entry into the healthcare field, and time working with HIVinfected patients was provided by 157 of $201 \mathrm{HCWs}$ who had a history of a positive tuberculin skin test. Of these, $25 \%$ tested positive before working in health care, $25 \%$ after entering health care but before working with HIV-infected patients, and the remaining $50 \%$ after starting to work with HIV-positive patients. The $805 \mathrm{HCWs}$ with no history of either TB or a positive skin test received a baseline PPD; 39 had new positive skin tests, and 766 had a negative PPD. Thus, of the 1,014 HCWs enrolled, a total of 248 (24\%) were defined as positive at study entry, with a history of active $\mathrm{TB}$, or a newly or previously positive PPD test.

The percentage of HCWs who were positive at study entry in various subgroups is given in Table 1. Several factors were significantly related to a higher rate of positivity, including BCG vaccination in the past. Factors that did not appear to have an impact included the percentage of the patient caseload who were known to be HIV-infected, years working with HIV-infected patients, and having direct or indirect contact with HIV-infected patients (counseling or educational sessions, scheduling appointments, etc).

Multiple logistic regression analyses were performed to identify factors that were independently related to being positive at study enrollment. The variables included were age, working in the NYC area, being foreign born or previously residing in a TB endemic area, BCG vaccination in the past, occupational type, years in the healthcare field, years in the HIV field, proportion of patients cared for with HIV, proportion of patients cared for with active TB, hours of direct hands-on care, and the performance of cough-inducing procedures including bronchoscopies, endoscopies, and the administration of aerosols.

Interactions of other variables with a history of BCG vaccination were suggested by both logistic regression and classification and regression tree analyses. Thus, the logistic regression was performed separately for those with and without a BCG vaccination. For the subset with a history of $B C G$ vaccination, no other variables were found to be significantly related to positivity at study entry. For those without a known history of BCG vaccination, three factors were significantly related to baseline positivity: working in the NYC area, being foreign-born or previously residing in a

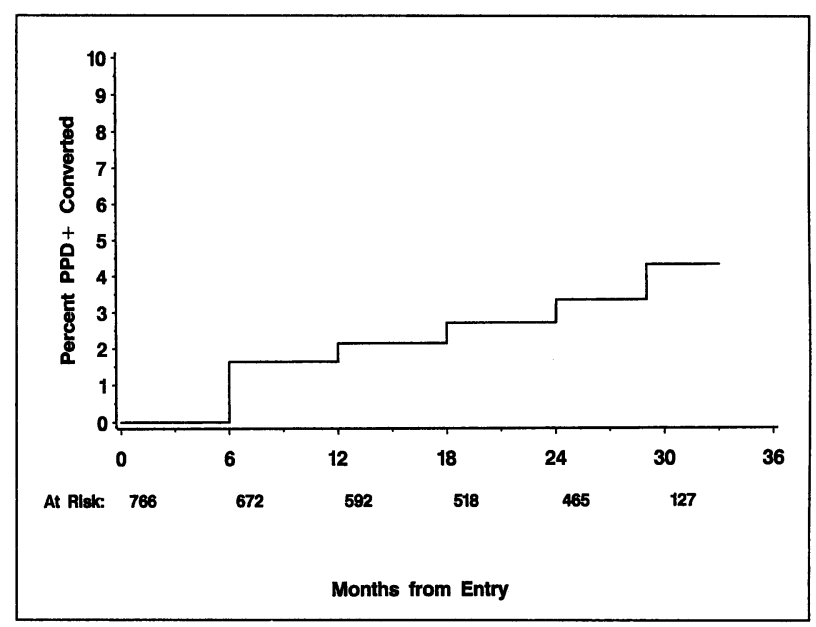

FIGURE. Kaplan-Meier curve of purified protein derivative conversion rate of 766 healthcare workers who were negative at baseline and retested every 6 months.

TB endemic area, and length of time as an HCW (Table 2). Age, providing direct patient care, performing coughinducing procedures, and working with HIV-infected patient populations were not found to be independent predictors of acquired TB or a positive skin test at baseline.

Of the $766 \mathrm{HCWs}$ who were followed in the prospective portion of the study with skin testing every 6 months, 376 were from the greater New York City area, and 390 came from the 10 other units. Twenty-six had a prior history of BCG vaccination, 73 had received two-step testing at baseline, and 13 had self-reported as being HIV-infected. The average length of follow-up was 19.6 months, with $66 \%$ receiving at least three follow-up skin tests. A total of 22 HCWs (3\%) converted their PPD or received TB prophylaxis during the course of the study, at a rate of 1.8 per 100 person years of follow-up. Kaplan-Meier estimates of PPD conversion are shown in the Figure. Of the initial cohort, $441(58 \%)$ attended a final study visit or were counted in the conversion group. Of the $325 \mathrm{HCWs}$ who missed the final visit, 244 (75.1\%) had moved or changed occupation during the course of the study.

There were no reported cases of active TB among the participating HCWs at any of the units during the study. Only one converter had reported a prior history of BCG vaccination, three had received two-step baseline testing, and none of the self-identified HIV-positive HCWs converted their PPD while participating in this study. Fourteen of the 22 skin-test conversions occurred in three units: one unit in NYC (5 conversions), Richmond (6 conversions), and New Orleans (3 conversions). None of these conversions were associated with each other through a known cluster of conversions after exposure to an index case of TB. Of 17 converters with no induration at baseline, 2 had follow-up indurations between 10 and $14 \mathrm{~mm}, 9$ had between 15 and $20 \mathrm{~mm}$, and 6 had indurations greater than $20 \mathrm{~mm}$. Two HCWs with baseline indurations between 5 and $9 \mathrm{~mm}$ had follow-up indurations between 10 and $14 \mathrm{~mm}$ and were assessed by unit staff as being converters. Three $\mathrm{HCWs}$ with no induration at baseline were counted as con- 
TABLE 3

Purified Protein Derrvative Conversion Rates by Occupational Risk Factors

\begin{tabular}{|c|c|c|c|c|c|}
\hline Occupatlonal Risk Factor & No. & PPD+ & Rate* & $\mathbf{C l}_{95}^{\dagger}$ & $\boldsymbol{P}$ \\
\hline \multicolumn{6}{|l|}{ Work in NYC } \\
\hline Yes & 376 & 9 & 1.6 & $(0.8-3.1)$ & \\
\hline No & 390 & 13 & 1.8 & $(1.0-3.1)$ & .80 \\
\hline \multicolumn{6}{|c|}{ Performs any cough-inducing procedures } \\
\hline Yes & 216 & 9 & 2.4 & $(1.1-4.5)$ & \\
\hline No & 550 & 13 & 1.5 & $(0.8-2.5)$ & .28 \\
\hline \multicolumn{6}{|l|}{ Occupation } \\
\hline $\mathrm{MD}, \mathrm{PA}, \mathrm{NP}$, or nurses & 430 & 11 & 1.7 & $(0.8-3.0)$ & \\
\hline SW, counselor, clerical, or other & 336 & 11 & 1.8 & $(0.9-3.3)$ & .88 \\
\hline \multicolumn{6}{|l|}{ Proportion of patients with HIV } \\
\hline $0 \%-49 \%$ & 234 & 6 & 1.7 & $(0.6-3.7)$ & \\
\hline $50 \%-100 \%$ & 440 & 14 & 1.8 & $(1.0-3.0)$ & .99 \\
\hline Unknown or N/A & 92 & 2 & 1.6 & $(0.2-5.8)$ & \\
\hline \multicolumn{6}{|l|}{ Proportion of patients with active TB } \\
\hline $0 \%-24 \%$ & 524 & 15 & 1.7 & $(1.0-2.8)$ & \\
\hline $25 \%-100 \%$ & 58 & 4 & 4.6 & $(1.2-11.7)$ & .15 \\
\hline Unknown or N/A & 184 & 3 & 1.0 & $(0.2-3.0)$ & \\
\hline \multicolumn{6}{|l|}{ Current occupational setting } \\
\hline Outpatient & 218 & 0 & 0.0 & $(0.0-1.1)$ & $<.0001$ \\
\hline Hospital-based & 394 & 13 & 1.9 & $(1.0-3.2)$ & \\
\hline Private practice & 77 & 4 & 3.2 & $(0.9-8.2)$ & \\
\hline Drug treatment program & 52 & 5 & 9.5 & $(3.1-22.1)$ & \\
\hline Other & 24 & 0 & 0.0 & $(0.0-9.7)$ & \\
\hline Overall & 766 & 22 & 1.8 & $(1.1-2.7)$ & \\
\hline
\end{tabular}

verters due to material exposure to cases of TB and subsequent prophylaxis. These three, per instructions in the protocol, did not receive further PPD testing, and thus it is unknown if they really were converters.

There was no apparent relation between caring for HIV-infected individuals and the rate of PPD conversion among our HCW cohort (Table 3). Those who performed cough-inducing procedures had tendencies toward higher conversion rates than those who didn't, but without apparent correlation to the HIV status of the patients. Working in settings with a higher proportion of patients with active TB appeared to increase the risk of acquiring TB infection, as did working in private practice or drug treatment programs. Using the variables in Table 3, a multivariate logistic analysis was performed to identify independent risk factors for PPD conversion. The only statistically significant finding associated with an increased risk of conversion was the occupational setting of a drug treatment program compared to all other settings (odds ratio, 4.4; $95 \%$ confidence interval, $1.5-12.3 ; P=.006)$.

\section{DISCUSSION}

As the most important opportunistic infection associated with HIV that can be transmitted to the general pop- ulation, TB warrants special concern among providers of health care and other services to HIV-infected populations. Multiple instances of patient-to-patient and patientto-HCW transmission of TB infection have been documented. ${ }^{3-7,14,15}$ Delays in diagnosing active cases of TB, plus antiquated and inadequate ventilation systems in certain hospitals and other healthcare facilities, contribute to the problem of TB transmission. ${ }^{11,16-18}$ In several cases of known exposure to an index case of TB, as many as $50 \%$ to $76 \%$ of HCWs had documented skin-test conversions. ${ }^{1,10}$ The majority of these reported cases of transmission have involved patients coinfected with HIV, leading to the presumption that working with HIV-infected populations may increase the risk to $\mathrm{HCWs}$ of acquiring TB infection. With HIV infection increasing among populations already known to be at risk for TB infection, ${ }^{19} \mathrm{HCWs}$ may encounter more patients with undiagnosed TB and may have a higher risk of becoming infected themselves. This is of special concern to the HIV-infected HCW, where the risk of acquisition of infection appears to be higher and the likelihood of progression to disease more certain. ${ }^{20}$

Our study included a large, diverse sample of 1,014 persons working in healthcare settings with HIV-infected clients. We identified four factors associated with being 
PPD-positive at study entry, including those listed in Table 2 , plus a history of BCG vaccination. Of greater interest are the factors not associated with baseline positivity, including working with HIV-infected populations in general, job category or description, amount and type of patient contact, and performing cough-inducing procedures such as sputum collection (although these procedures were done by only a small number of the HCWs in our study). Our baseline data reflect lifetime exposure, including cumulative time in health care, as well as exposure from the community. Some studies have found a correlation between TB infection and lower socioeconomic status that is stronger than the risk of infection through occupational exposure. ${ }^{21,22}$ It also may be that length of time as a HCW is a surrogate marker for age. These data are supported by the work of Bailey et al, ${ }^{22}$ who found that stratifying $\mathrm{HCWs}$ based on the amount of patient contact was not useful in determining the risk of previously acquired TB infection.

A study done at a New York City hospital identified the same independent predictors of a reactive tuberculin skin test among new employees hired between 1991 and 1993..$^{23}$ There was a baseline prevalence of $40 \%$ among these employees, which is higher than but comparable to our NYC rate of $31 \%$. Another NYC hospital reported similar findings, with a baseline prevalence of $45 \%$ in $1991 .{ }^{24}$

During the 33-month follow-up period for this study, we noted a PPD conversion rate of 1.8 per 100 person years, with a total of $19(2.5 \%) \mathrm{HCWs}$ having skin tests being reported as newly reactive and 3 others $(0.5 \%)$ who received TB prophylaxis and were counted as converters. Their inclusion may make the conversion rate slightly overestimated. Had they not been included, the rate would have been 1.6 per 100 person years. National rates of PPD conversion for HCWs in non-outbreak, non-HIV-specific settings is reported to be $<2 \%$ per year, ${ }^{20,25}$ which is comparable to our results. A Centers for Disease Control and Prevention (CDC) study of HCWs in Boston and NYC reported a conversion rate of $1.4 \%$ for participants who had received at least one follow-up skin test, scheduled every 6 months. ${ }^{26}$ "High-risk" HCWs from the 1993 SHEA-CDC TB Survey had an average annual conversion rate of $1.2 \%$, with a maximum rate of $2.8 \%$ at hospitals with less stringent TB control measures. ${ }^{27}$

Rates of skin-test conversion have been reported from individual hospitals. At a Canadian hospital, Liss and colleagues found a somewhat lower overall conversion rate of 0.86 per 100 person years. ${ }^{28}$ However, those HCWs with greater exposure to patients with active TB had a higher proportion of PPD conversions, up to 5\% over the 42-month study period for emergency room staff. Another study from a hospital in NYC documented a 3.1\% overall skin-test conversion rate during an 18-month pre-intervention period from January 1990 to June 1991, with higher rates for those with more direct patient contact (6.4\% vs $1.0 \%) .{ }^{29}$ However, after instituting additional transmission control measures for the intervention period of July 1991 to August 1992, conversion rates for these two general groups were similar (4.7\% vs $2.3 \%$ ), while the overall conversion rate remained the same at 3.0\%. There also were no significant differences between those assigned to areas with higher proportions of patients with TB or HIV and those assigned to other areas.

Our results suggest that working with HIV-infected populations, providing direct medical or nursing care, or working in the NYC area does not increase an individual's risk of acquiring TB infection. Our conversion rates for different occupational categories (direct patient-care jobs vs other patient contact) were 1.7 and 1.8 per 100 person years respectively, significantly less than the average rate of 3.3 in 1993 to 1994 reported by a NYC hospital with a high TB case rate. ${ }^{24}$ Our data show a trend toward higher conversion rates for HCWs who cared for a greater proportion of patients known to have active TB or who performed coughinducing procedures and therefore were more likely to be exposed to aerosolized infectious particles. The apparently higher risk for those working in private practice may reflect less stringent infection control measures and a lower index of suspicion for TB. Many drug treatment programs and methadone clinics do not provide medical care on-site, possibly contributing to a delay in the assessment of symptoms suggestive of TB.

We attempted to evaluate the possible impact of the HIV status of the HCWs through the anonymous questionnaires, which were included to assist in the interpretation of PPD results. Castro and Dooley ${ }^{30}$ also note that HIV infection greatly increases an HCW's risk of developing active TB, if infected with Mycobacterium tuberculosis, thereby causing an apparent difference when evaluating relative risk. We noted the reluctance of some HCWs to provide HIV status information even in an anonymous format (26\%) and also that many providers working in HIV care did not know their own serostatus (28\%). Few participants (2\%) reported being HIV-infected, however, so this information about HIV status and its effect on the interpretation of PPD results and conversion rates must be interpreted cautiously. Only 10 other participants reported a PPD induration of 5 to $10 \mathrm{~mm}$ that was classified as a nonconversion during the study follow-up period. Even if all 10 were HIV-positive and should have been classified as PPD conversions, this would only increase our total conversions from $3 \%$ to $4 \%$, with an event rate of 2.6 per 100 person years of follow-up.

One limitation of this study was the difficulty in identifying a valid external control group to determine the difference in risk between HCWs caring for HIV-infected patients and HCWs caring for the general population. We were not able to collect data on the PPD status of other HCWs involved in non-HIV care within each participating unit. For this reason, comparisons were made between different geographical areas, amount and type of patient contact, degree of potential exposure to $T B$, and other demographic factors. Within our cohort, we found no significant relation between the proportion of patients cared for who were HIV-positive and subsequent PPD conversion. Because the number of conversions was small, however, such a relation may have been missed. Another limitation was that each unit requested the autonomy to assess TB risk for their HCWs and to 
make medical decisions about conversion and the need for prophylaxis. This may have led to the inclusion of up to five HCWs who were not true converters, but it does indicate community practice in TB risk assessment.

Our data provide some reassurance that caring for HIV-infected patients in itself is not an independent source of increased risk of TB infection in HCWs. A high index of suspicion for TB when evaluating all patients, rigorous compliance with infection control guidelines, and careful follow-up to ensure compliance with treatment regimens should minimize the potential for infection with TB. ${ }^{31,32}$ An awareness among HCWs that the incidence of TB among HIV-infected clients is high relative to noninfected clients, balanced against our data showing no independent increased prevalence or incidence of TB infection in those who work directly with HIV-infected patients, should assist HCWs in protecting themselves without undue fear of the HIV-infected patient. The greater risk for acquiring TB infection appears to be related to the prevalence of the disease in the community and in the overall patient population, and not specifically to occupational exposure to HIV-infected patients. ${ }^{22}$

\section{REFERENCES}

1. Sepkowitz KA. Tuberculosis and the health care worker: a historical perspective. Ann Intern Med 1994;120:71-79.

2. Menzies D, Fanning A, Yuan L, Fitzgerald M. Tuberculosis among health care workers. N Engl J Med 1995;332:92-97.

3. Centers for Disease Control. Transmission of multidrug-resistant tuberculosis among immunocompromised persons in a correctional systemNew York, 1991. MMWR 1992;41:507-509.

4. Beck-Sague C, Dooley SW, Hutton MD, Otten J, Breeden A, Crawford JT, et al. Hospital outbreak of multi-drug resistant Mycobacterium tuberculosis infections. JAMA 1992;268:1280-1286.

5. Pearson ML, Jereb JA, Frieden TR, Crawford JT, Davis BJ, Dooley SW, et al. Nosocomial transmission of multidrug-resistant Mycobacterium tuberculosis. Ann Intern Med 1992;117:191-196.

6. Dooley SW, Villarino ME, Lawrence M, Salinas L, Amil S, Rullan JV, et al. Nosocomial transmission of tuberculosis in a hospital unit for HIVinfected patients. JAMA 1992;267:2632-2635

7. DiPerri G, Cadeo GP, Castelli F, Micciolo R, Basseti S, Rubini F, et al. Transmission of HIV-associated tuberculosis to healthcare workers. Infect Control Hosp Epidemiol 1993;14:67-72.

8. Barnes PF, Bloch AB, Davidson PT, Snider DE. Tuberculosis in patients with human immunodeficiency virus infection. $N$ Engl $\mathrm{J} \mathrm{Med}$ 1991;324:1644-1650.

9. Wurtz R, Fernandez J, Jovanovic B. Real and apparent tuberculin skin test conversions in a group of medical students. Infect Control Hosp Epidemiol 1994;15:516-519.

10. Griffith DE, Hardeman JL, Zhang Y, Wallace RJ, Mazurek GH. Tuberculosis outbreak among healthcare workers in a community hospital. Am J Respir Crit Care Med 1995;152:808-811.

11. Blumberg HM, Watkins DL, Berschling JD, Antle A, Moore P, White N, et al. Preventing the nosocomial transmission of tuberculosis. Ann Intern Med 1995;122:658-663.

12. American Thoracic Society. Diagnostic standards and classification of tuberculosis. Am Rev Respir Dis 1990;142:725-735.

13. Breiman L, Friedman JH, Olshen RA, Stone CJ. Classification and Regression Trees. Belmont, CA: Wadsworth; 1984.

14. Fischl MA, Uttamchandani RB, Daikos GL, Poblete RB, Moreno JN, Reyes RR, et al. An outbreak of tuberculosis caused by multiple-drugresistant tubercle bacilli among patients with HIV infection. Ann Intern Med 1992;117:177-183.

15. Edlin BR, Tokars JI, Grieco MH, Crawford JT, Williams J, Sordillo EM, et al. An outbreak of multidrug-resistant tuberculosis among hospitalized patients with the acquired immunodeficiency syndrome. $N$ Engl J Med 1992;326:1514-1521.

16. Kramer F, Modilevsky T, Waliany AR, Leedom JM, Barnes PF. Delayed diagnosis of tuberculosis in patients with human immunodeficiency virus infection. Am J Med 1990;89:451-456.

17. Hopewell PC. Impact of human immunodeficiency virus infection on the epidemiology, clinical features, management, and control of tuberculosis. Clin Infect Dis 1992;15:540-547.

18. Centers for Disease Control. Nosocomial transmission of multidrugresistant tuberculosis among HIV-infected persons-Florida and New York, 1988-1991. MMWR 1991;40:585-591.

19. Cantwell MF, Snider DE, Cauthen GM, Onorato IM. Epidemiology of tuberculosis in the United States, 1985 through 1992. JAMA 1994;272:535-539.

20. Sepkowitz KA. AIDS, tuberculosis, and the health care worker. Clin Infect Dis 1995; 20:232-242.

21. McKenna MT, Hutton M, Cauthen G, Onorato IM. The association between occupation and tuberculosis: a population-based survey. $A m \mathrm{~J}$ Respir Crit Care Med 1996;154:587-593.

22. Bailey TC, Fraser VJ, Spitznagel EL, Dunagan WC. Risk factors for a positive tuberculin skin test among employees of an urban, Midwestern teaching hospital. Ann Intern Med 1995;122:580-585.

23. Sepkowitz KA, Fella P, Rivera P, Villa N, DeHovitz J. Prevalence of PPD positivity among new employees at a hospital in New York City. Infect Control Hosp Epidemiol 1995;16:344-347.

24. Louther J, Rivera P, Feldman J, Villa N, DeHovitz J, Sepkowitz KA. Risk of tuberculin conversion according to occupation among health care workers at a New York City hospital. Am J Respir Crit Care Med 1997;156:201-205.

25. Fridkin SK, Manangan L, Bolyard E, Jarvis WR. SHEA-CDC TB survey, part I: status of TB infection control programs at member hospitals, 1989-1992. Infect Control Hosp Epidemiol 1995;16:129-134.

26. Panlilio A, Burwen D, the TB Infection Surveillance Project, Centers for Disease Control and Prevention. Tuberculosis (TB) skin testing surveillance of health care workers (HCWs). Presented at the Sixth Annual Meeting of the Society for Healthcare Epidemiology of America; April 21-23, 1996; Washington, DC. Abstract 12.

27. Fridkin SK, Manangan L, Bolyard E, and Jarvis WR. SHEA-CDC TB survey, part II: efficacy of TB infection control programs at member hospitals, 1992. Infect Control Hosp Epidemiol 1995;16:135-140.

28. Liss GM, Khan R, Koven E, Simor AE. Tuberculosis infection among staff at a Canadian community hospital. Infect Control Hosp Epidemiol 1996;17:29-35.

29. Maloney SA, Pearson ML, Gordon MT, Del Castillo R, Boyle JF, Jarvis WR. Efficacy of control measures in preventing nosocomial transmission of multidrug-resistant tuberculosis to patients and health care workers. Ann Intern Med 1995;122:90-95.

30. Castro KG, Dooley SW. Mycobacterium tuberculosis transmission in healthcare settings: is it influenced by coinfection with human immunodeficiency virus? Infect Control Hosp Epidemiol 1993;14:65-66.

31. American Thoracic Society. Control of tuberculosis in the United States. Am Rev Respir Dis 1992;146:1623-1633.

32. Centers for Disease Control. Guidelines for preventing the transmission of Mycobacterium tuberculosis in health-care facilities, 1994. MMWR 1994;43:RR13. 